\title{
STUDY OF CALCULATION METHOD OF PURE MODE II STRESS INTENSITY FACTOR OF FINE-GRAINED CONCRETE USING DIFFERENT NUMERICAL MODELS
}

\author{
Changlin Zhou \\ Chengdu Surveying Geotechnical Research Institute Co., Ltd. of MCC, Chengdu, China; and \\ State Key Laboratory of Hydraulics and Mountain River Engineering, School of Architecture and Environment, \\ Sichuan University, Chengdu, China; and \\ Key Laboratory of Deep Underground Science and Engineering (Ministry of Education), School of Architecture \\ and Environment, Sichuan University, Chengdu, China
}

Bo Peng, An Deng, Xiaofeng Gao, Yaojia Li

Chengdu Surveying Geotechnical Research Institute Co., Ltd. of MCC, Chengdu, China

Lei Zhou, Zheming Zhu

State Key Laboratory of Hydraulics and Mountain River Engineering, School of Architecture and Environment, Sichuan University, Chengdu, China; and

Key Laboratory of Deep Underground Science and Engineering (Ministry of Education), School of Architecture and Environment, Sichuan University, Chengdu, China

e-mail:zhouleittkx@scu.edu.cn; zhuzheming@scu.edu.cn

\begin{abstract}
Measuring and calculating methods of critical stress intensity factors (SIFs) have become hot topics which attracted large attention recently. In this work, anti-symmetrical four-point bending tests of cracked fine-grained concrete specimens were conducted experimentally and numerically by using a computer-controlled universal testing machine and ABAQUS code. A comparative study of the calculation method of pure mode II stress intensity factor of a fine-grained concrete was performed by utilizing the conventional finite element method (FEM) in two and three dimensions as well as the extended finite element method (XFEM) in three dimensions. The results show that in three-dimensional models, the crack mode is closest to the pure mode II at the center of specimen thickness. Pure mode II stress intensity factors obtained by SEAM ${ }^{2 \mathrm{D}}$ and $\mathrm{XFEM}^{3 \mathrm{D}}$ are 1.013 and 1.0617 times that by $\mathrm{SEAM}^{3 \mathrm{D}}$, respectively. Pure mode II stress intensity factors of the fine-grained concrete obtained by the conventional FEM are more stable than that by XFEM. The number of mesh circles has slight influence on the calculation results of pure mode II stress intensity factor.
\end{abstract}

Keywords: fine-grained concrete, critical stress intensity factor, $J$-integral, anti-symmetrical four-point bending, FEM, XFEM

\section{Introduction}

Since Kaplan (1961) introduced fracture mechanics into concrete in 1961, fracture mechanics of concrete has attracted large attention. Also, how to apply fracture mechanics to concrete structures has been investigated by many researchers. Fracture mechanics of concrete has been widely applied in construction engineering, and a remarkable progress has been obtained. In fracture mechanics, the crucial issue is to analyze the stress and strain field in the vicinity of the crack tip, and to obtain dominant parameters that control crack behavior, aiming at establishing a reasonable fracture criterion.

As the stress intensity factor (SIF) exceeds the threshold value, i.e. the fracture toughness, the crack will initiate and propagate, which could induce large engineering disasters. Therefore, 
determination of SIF has become an important issue in fracture mechanics. Therefore, the study of the stress intensity factor is of great significance in the engineering.

Currently, many scholars have conducted a great deal of research on the stress intensity factors of concrete. Braham and Buttlar (2009) investigated pure mode II fracture resistance of asphalt concrete by performing an anti-symmetrical four-point loading test on the beam specimen. Fett (1999) applied a weight function and a superposition method to solve the stress intensity factor of pre-cracking plates under bending-tensile load. Golewski et al. (2012) used the extended finite element method to simulate crack growth of concrete containing siliceous fly ash under pure mode II fracturing. Dong et al. (2018) conducted a comparative study on utilizing the stress intensity factor-based criterion to predict the mixed mode I-II crack propagation in concrete. Wu et al. (2017) developed a flexibility determination method based on numerical simulation to calculate the stress intensity factor and fracture toughness of concrete. Orowan (1949) and Irwin (1957) claimed that there was a particular relationship between the energy release ratio and stress intensity factor. Stys and Minch (1993) investigated the effect of mode II stress intensity factor on fracture toughness by using a photo-elastic coating method for concrete. However, less research is reported on performing a study on calculating pure mode II stress intensity factor of fine-grained concrete under different numerical models by using ABAQUS code.

The stress intensity factor $K$ is usually applied to demonstrate the crack field intensity in linear elastic fracture mechanics analysis in engineering applications. The methods of determining stress intensity factors include analytical methods, numerical methods and experimental methods. The analytical method can be used only to calculate simple problems, but most problems need numerical solution to work out. Nowadays, the finite element method is widely employed in the numerical solution of engineering problems, and how to solve the stress intensity factor in the engineering has become more and more important. However, there is less research on the study of pure mode II stress intensity factor of fine-grained concrete. In this paper, antisymmetrical four-point bending tests of a cracked fine-grained concrete specimen were carried out by using a universal testing machine, whose stress intensity factors were computed with a formula and ABAQUS code. Additionally, a comparative study of calculating pure mode II stress intensity factor of fine-grained concrete was performed by utilizing the conventional finite element method (FEM) in two and three dimensions, and the extended finite element method (XFEM) (ABAQUS, 2016) in three dimensions. Furthermore, variation of the stress intensity factor of concrete in the thickness direction and on different contours was studied. Also, the effect of mesh circles in the vicinity of the crack tip on calculating SIF was investigated.

\section{Experimentation}

In order to obtain pure mode II fracture toughness (critical stress intensity factor) of fine-grained concrete, tests of anti-symmetrical four-point bending (Wang et al., 2016) were conducted by using cracked fine-grained concrete specimens and ABAQUS code.

\subsection{Materials}

Ordinary Portland Cement (OPC) with a strength grade of $42.5 \mathrm{MPa}$ was utilized in this experiment, and chemical and mineral composition as well as physical-mechanical parameters are shown in Table 1-3. In the light of coarse aggregate blocking crack growth (Zhou et al., 2018, 2019b; Zhou and Zhu, 2019a) and increasing the dispersion of test results, fine river sand was employed in the experimentation. 
Table 1. Chemical composition of cement

\begin{tabular}{|c|c|c|c|c|c|c|c|c|c|}
\hline Chemical composition & $\mathrm{SiO}_{2}$ & $\mathrm{Al}_{2} \mathrm{O}_{3}$ & $\mathrm{CaO}$ & $\mathrm{Fe}_{2} \mathrm{O}_{3}$ & $\mathrm{MgO}$ & $\mathrm{K}_{2} \mathrm{O}$ & $\mathrm{SO}_{3}$ & $\mathrm{TiO}_{2}$ & LOI* \\
\hline \hline Cement [\%] & 18.63 & 2.49 & 71.7 & 2.62 & 3.24 & 0.48 & 5.00 & 0.84 & 4.75 \\
\hline
\end{tabular}

Table 2. Mineral composition of cement

\begin{tabular}{|c|c|c|c|c|c|}
\hline Mineral composition & $\mathrm{Ca}_{3} \mathrm{SiO}_{5}$ & $\mathrm{Ca}_{2} \mathrm{SiO}_{4}$ & $\mathrm{Ca}_{2} \mathrm{Fe}_{1.40} \mathrm{Al}_{0.60} \mathrm{O}_{5}$ & $\mathrm{CaSO}_{4}$ & $\mathrm{CaCO}_{3}$ \\
\hline \hline SemiQuant [\%] & 45 & 39 & 4 & 7 & 6 \\
\hline
\end{tabular}

Table 3. Physical and mechanical properties of cement

\begin{tabular}{|c|c|c|c|c|c|c|c|}
\hline \multirow{2}{*}{ Soundness } & \multicolumn{2}{|c|}{$\begin{array}{c}\text { Setting time } \\
{[\mathrm{min}]}\end{array}$} & \multicolumn{2}{|c|}{$\begin{array}{c}\text { Flexural strength } \\
{[\mathrm{MPa}]}\end{array}$} & \multicolumn{2}{|c|}{$\begin{array}{c}\text { Compressive } \\
\text { strength }[\mathrm{MPa}]\end{array}$} & $\begin{array}{c}\text { Specific } \\
\text { area } S_{b} \\
{\left[\mathrm{~m}^{2} / \mathrm{kg}\right]}\end{array}$ \\
\cline { 2 - 7 } & Initial & Final & $3 d$ & $28 d$ & $3 d$ & $28 d$ & 360 \\
\hline \hline Pass & 170 & 210 & 6.0 & 7.9 & 25.3 & 51.3 & 360 \\
\hline
\end{tabular}

\subsection{Specimen geometry and preparation}

Fine-grained concrete was applied to fabricate specimens in this experiment, and the mix proportion of cement, sand and water was 1:3.5:0.5, respectively, by mass. Initially, river sand and cement were sent into a concrete mixer, which were stirred for about $5 \mathrm{~min}$. Then a certain amount of water was added into the mixtures which were stirred for about $5 \mathrm{~min}$. Subsequently, rectangular plastic moulds, having the internal dimension of $100 \mathrm{~mm} \times 100 \mathrm{~mm} \times 400 \mathrm{~mm}$, were filled with concrete, then were vibrated on a shaking table for 40 seconds to eliminate bubbles in concrete. After $24 \mathrm{~h}$, the moulds were removed, and the concrete specimens were cured in the water tank for 28 days at room temperature. Finally, the specimens were taken out from the water tank and cut with a marble cutter to obtain the initial precrack as illustrated in Fig. 1, whose depth was set as $30 \mathrm{~mm}$. The dimension and loading of the anti-symmetrical four-point bending specimen is shown in Fig. 2.

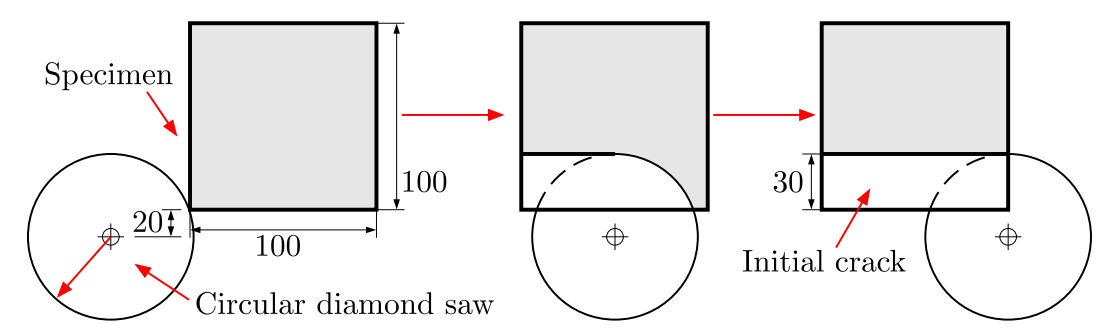

Fig. 1. Schematic diagram of the pre-crack cutting procedure for ASFPB specimen [mm]

The anti-symmetrical four-point bending tests were carried out by a universal testing machine whose loading rate was controlled by the displacement. In the light of a vast array of in-situ tests and related literature (Wittmann et al., 1987; Zhang et al., 2009), the loading rate was set as $0.05 \mathrm{~mm} / \mathrm{min}$ to make the precrack in fine-grained concrete propagate steadily.

The load distribution is mainly determined by $S_{1}$ and $S_{2}$ as shown in Fig. 2. Only when appropriate $S_{1}$ and $S_{2}$ are chosen, pure mode II fracture can occur. Referring to the four-point bending test in (Zhang et al., 2009), $S_{1}$ and $S_{2}$ were designed as $150 \mathrm{~mm}$ and $30 \mathrm{~mm}$, respectively, and the fracture modes occurred as shown in Fig. 3. 
(a)
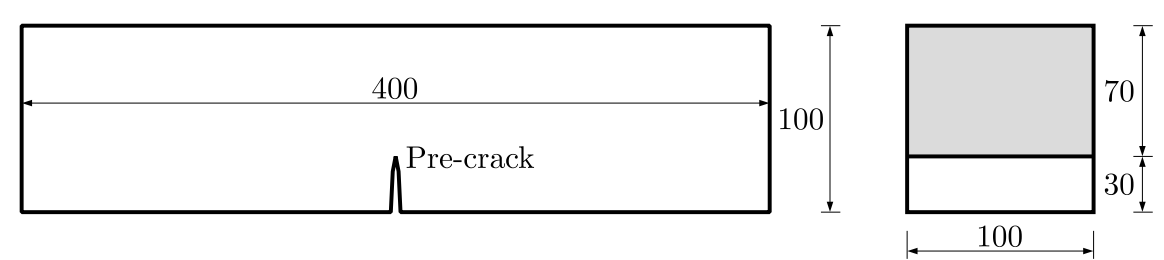

(b)

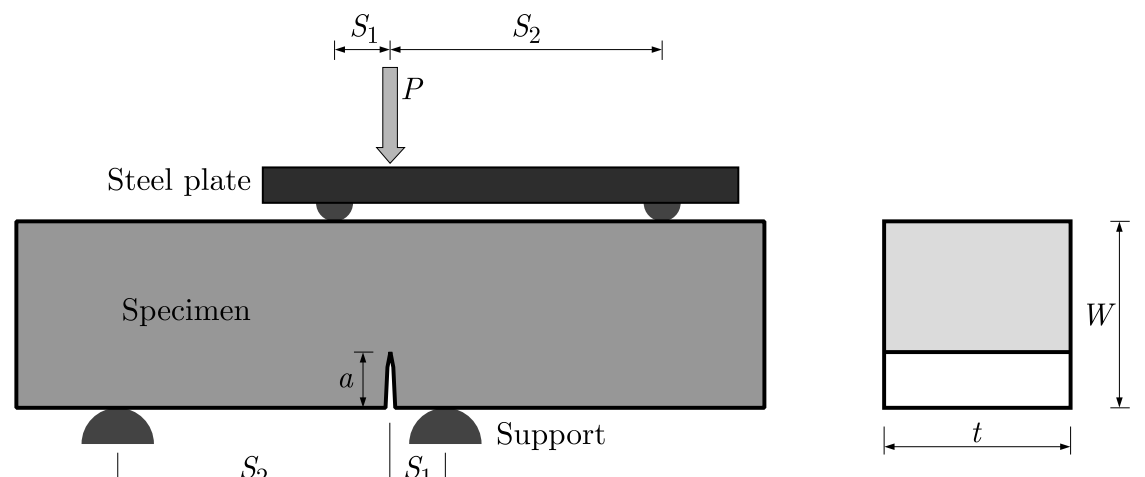

Fig. 2. Specimen dimensions and loading conditions [mm]: (a) dimensions, (b) loading of the concrete ASFPB specimen

(a)

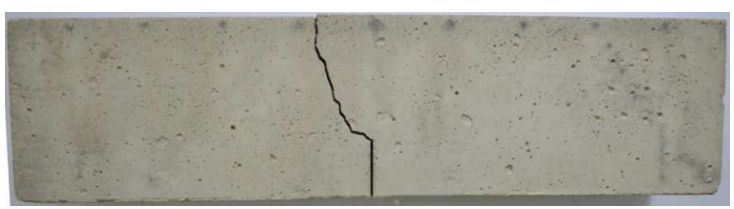

(c)

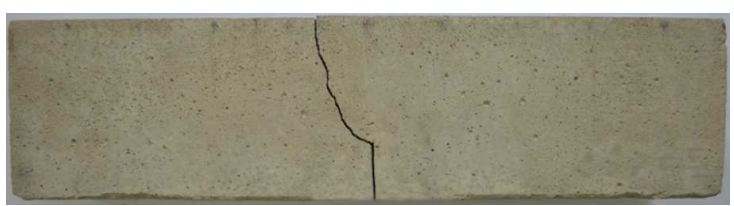

(b)

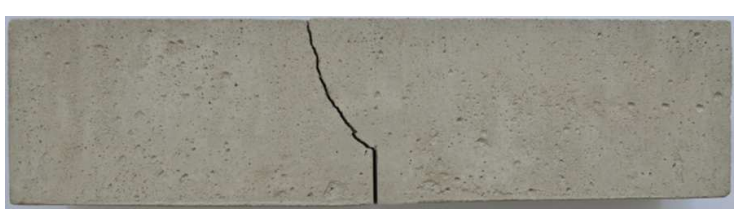

(d)

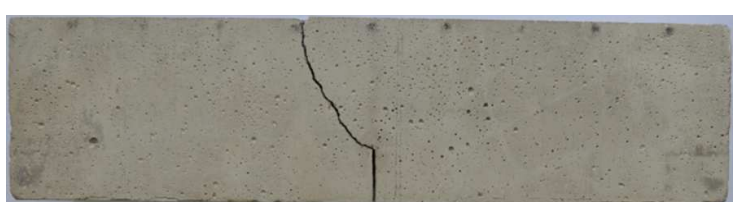

Fig. 3. Fracture mode of concrete ASFPB specimens: (a) 1\# specimen, (b) 2\# specimen, (c) 3\# specimen, (d) 4\# specimen

\section{Introduction of calculation methods of SIFs}

According to He et al. (1990), the stress intensity factor of anti-symmetrical four-point bending (ASFPB) specimens can be calculated by

$$
K_{I I}=\frac{Q \sqrt{\pi a}}{B t} Y_{I I}
$$

where $Q$ is the shearing force at the crack tip, $Q=\left(S_{2}-S_{1}\right) P /\left(S_{2}+S_{1}\right) ; P$ is the external load; $B, t$ and $a$ denote width, thickness and crack length of the model, respectively; $Y_{I I}$ is a normalized mode II SIF, also known as the shape factor, and $Y_{I I}$ is a function of the relative crack length $a / W$, which can be calculated by ABAQUS code based on Rice's $J$-integral (Rice, 1968).

\section{1. $J$-integral in two dimensions}

Two-dimensional $J$-integral is defined in quasi-static analysis as (ABAQUS, 2016)

$$
J=\lim _{\Gamma \rightarrow 0} \int_{\Gamma} \mathbf{n H q} d \Gamma
$$


where $\Gamma$ is a contour which is initiating from one crack surface and finishing on another crack surface, as illustrated in Fig. 4; $\mathbf{q}$ is the unit vector in the direction of virtual crack propagation; $\mathbf{n}$ is the outward normal of the contour $\Gamma$. $\mathbf{H}$ is given by

$$
\mathbf{H}=W \mathbf{I}-\boldsymbol{\sigma} \frac{\partial \mathbf{u}}{\partial u}
$$

where $W$ denotes elastic strain energy for elastic material behavior, and is defined as elastic strain energy density plus plastic dissipation for elastic-plastic or elastic-viscoplastic material behavior.

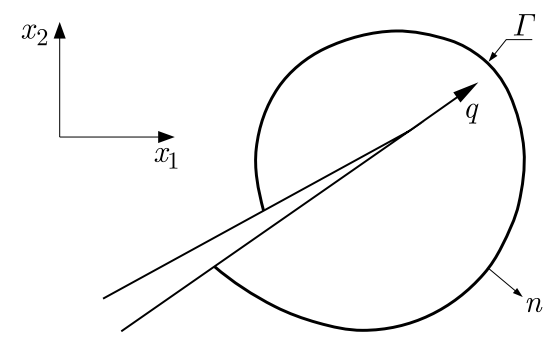

Fig. 4. Contour plot of the evaluating $J$-integral

Referring to Shih et al. (1986), Eq. (3.2) can be rewritten as

$$
J=-\oint_{C+C_{+}+\Gamma+C_{-}} \mathbf{m H} \overline{\mathbf{q}} d \Gamma-\int_{C_{+}+C_{-}} \mathbf{t} \frac{\partial \mathbf{u}}{\partial x} \overline{\mathbf{q}} d \Gamma
$$

where $\overline{\mathbf{q}}$ is a plentifully smooth weight function in the domain surrounded by the closed contour, and $\mathbf{m}$ is the outward normal of the region surrounded by the closed contour, as illustrated in Fig. $5 . \mathbf{m}=-\mathbf{n}$ on $\Gamma$, and $\mathbf{t}=\mathbf{m} \boldsymbol{\sigma}$ is the surface traction on $C_{+}$and $C_{-}$.

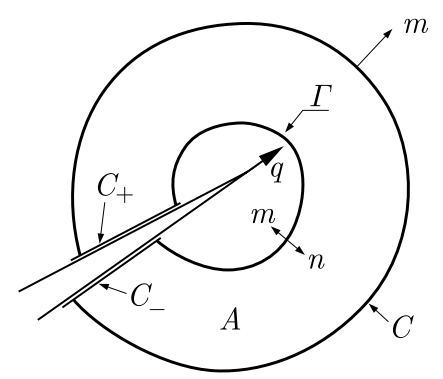

Fig. 5. The region $A$ is surrounded by the enclosed contour $C+C_{+}+\Gamma+C_{-}$

The enclosed contour integral is converted into the domain integral by utilizing the divergence theorem, namely

$$
J=-\int_{A} \frac{\partial}{\partial x}(\mathbf{H} \overline{\mathbf{q}}) d \Gamma-\int_{C_{+}+C_{-}} \mathbf{t} \frac{\partial \mathbf{u}}{\partial x} \overline{\mathbf{q}} d \Gamma
$$

where $A$ is the region surrounded by the enclosed contour $C+C_{+}+\Gamma+C_{-}$.

Supposing that the balance is satisfied, $W$ is a function of the mechanical strain, namely, $W=W\left(\varepsilon^{m}\right)$. Then, we obtain

$$
\frac{\partial}{\partial x} \boldsymbol{\sigma}+\mathbf{f}=\mathbf{0} \quad \frac{\partial W}{\partial x}=\frac{\partial W}{\partial \varepsilon^{m}} \frac{\partial \varepsilon^{m}}{\partial x}=\boldsymbol{\sigma}\left(\frac{\partial \varepsilon}{\partial x}-\frac{\partial \varepsilon^{t h}}{\partial x}\right)
$$

where $\mathbf{f}$ and $\varepsilon^{t h}$ denote the body force per unit volume and the thermal strain, respectively. 
Substituting Eqs. (3.6) into Eq. (3.5) gives

$$
J=-\int_{A}\left[\mathbf{H} \frac{\partial \overline{\mathbf{q}}}{\partial x}+\left(\mathbf{f} \frac{\partial \mathbf{u}}{\partial x}-\boldsymbol{\sigma} \frac{\partial \varepsilon^{t h}}{\partial x}\right) \overline{\mathbf{q}}\right] d \Gamma-\int_{C_{+}+C_{-}} \mathbf{t} \frac{\partial \mathbf{u}}{\partial x} \overline{\mathbf{q}} d \Gamma
$$

\section{2. $J$-integral in three dimensions}

Taking a tangential continuous front of the crack into account, the two-dimensional $J$-integral could be expanded to three dimensions, as illustrated in Fig. 6. The local direction of fictitious crack growth is still determined by $\mathbf{q}$, which is perpendicular to the local crack front and is located on the crack plane.

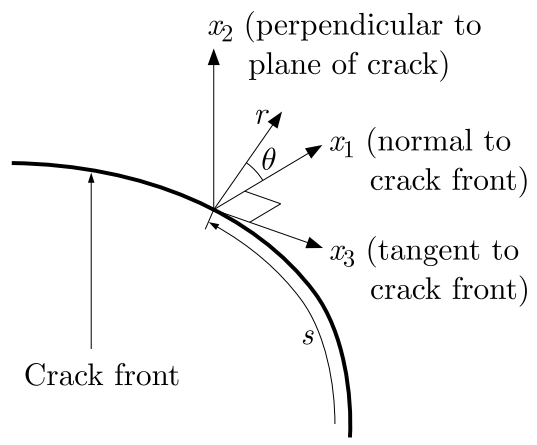

Fig. 6. Local orthogonal Cartesian coordinates defined at $s$ point of the crack front

Approximately, as $r$ tends towards 0 , the states of path independence can be applicable to any contour on the $x_{1}-x_{2}$ plane, which is perpendicular to the crack front at the point $s$. Therefore, the $J$-integral defined on this plane could be expanded to a pointwise energy release rate along the crack front as (ABAQUS, 2016)

$$
J(s)=\lim _{\Gamma \rightarrow 0} \int_{\Gamma} \mathbf{n H q} d \Gamma
$$

For a fictitious crack advance $\lambda(s)$ on the three-dimensional crack plane, the energy release rate can be expressed as

$$
\bar{J}=\lim _{L} J(s) \lambda(s) d s=\lim _{\Gamma \rightarrow 0} \int_{A_{t}} \mathbf{n H q} d A
$$

where $L$ is the considered crack front; $d A$ denotes an area element on a vanishingly small tubular surface surrounding the crack tip (namely, $d A=d s d \Gamma$ ); $\mathbf{n}$ represents the outward normal of $d A$.

$\bar{J}$ could be computed by utilizing the region integral method like that applied in two dimensions. To do so, the surface integral in Eq. (3.9) is converted into a volume integral by introducing a contour domain $A_{0}$, outside the domain $A_{t}$, external domain $A_{\text {ends }}$ with which the crack front ends, and the crack surfaces $A_{\text {cracks }}$, as illustrated in Fig. 7.

A weight function $\overline{\mathbf{q}}$ is introduced, whose magnitude is 0 on the surface $A_{0}$ and is $\lambda(s) \mathbf{q}$ on the surface $A_{t} . \overline{\mathbf{q}}$ is supposed to vary smoothly between these values in the region $A$. $\mathbf{q}$ on $A_{\text {ends }}$ is not tangential to the surface, which must be made so. This can be done in the ABAQUS code by explicitly defining the surface normals. Subsequently, Eq. (3.9) can be rewritten as

$$
\bar{J}=-\oint_{A} \mathbf{m H} \overline{\mathbf{q}} d A-\int_{A_{\text {ends }}+A_{\text {cracks }}} \mathbf{t} \frac{\partial \mathbf{u}}{\partial x} \overline{\mathbf{q}} d A
$$




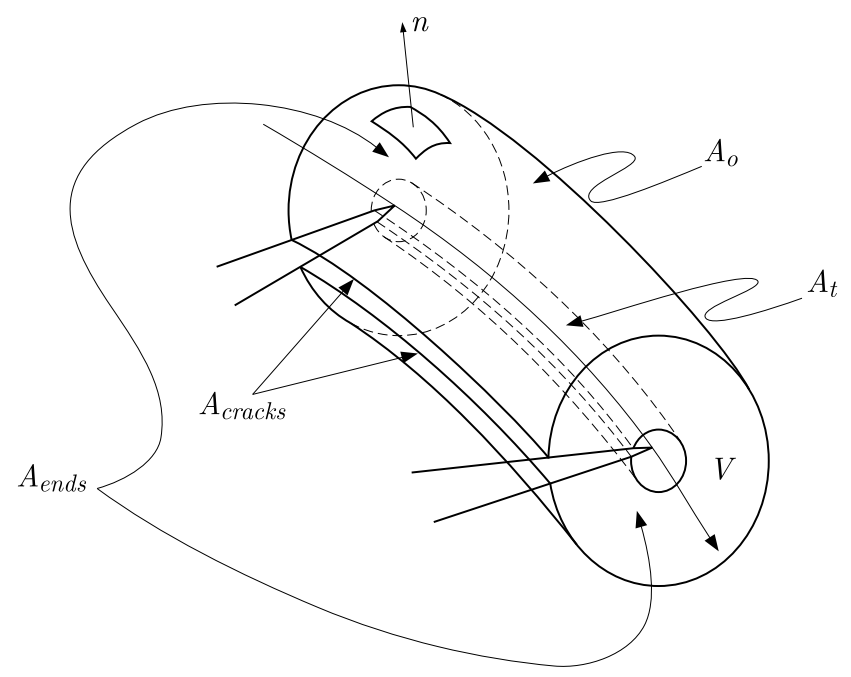

Fig. 7. The volume domain $V$ is enclosed by region $A=A_{t}+A_{0}+A_{\text {ends }}+A_{\text {cracks }}$

where $\mathbf{m}$ is the outward normal to the domain $A . \mathbf{t}=\mathbf{m} \boldsymbol{\sigma}$ is surface traction on the regions $A_{\text {ends }}$ and $A_{\text {cracks. }}$.

In the light of the divergence theorem, we have

$$
\bar{J}=-\int_{V}\left[\mathbf{H} \frac{\partial \overline{\mathbf{q}}}{\partial x}+\left(\mathbf{f} \frac{\partial \mathbf{u}}{\partial x}-\boldsymbol{\sigma} \frac{\partial \varepsilon^{t h}}{\partial x}\right) \overline{\mathbf{q}}\right] d V-\int_{A_{\text {ends }}+A_{\text {cracks }}} \mathbf{t} \frac{\partial \mathbf{u}}{\partial x} \overline{\mathbf{q}} d A
$$

In order to calculate $J(s)$ at each node set $P$ along the crack front line, the interpolation functions like those utilized in the finite elements are applied to discretize $\lambda(s)$

$$
\lambda(s)=N^{Q}(s) \lambda^{Q}
$$

where $\lambda^{Q}=1$ at $P$ and all other $\lambda^{Q}$ are equal to 0 .

Substituting Eq. (3.12) into Eq. (3.11) gives

$$
J^{P}=\frac{\bar{J}^{P}}{\int_{L} N^{P} d s}
$$

\section{Model meshing}

In this paper, the calculation of the stress intensity factor is investigated. Because the specimens have large dimensions $(100 \mathrm{~mm} \times 100 \mathrm{~mm} \times 400 \mathrm{~mm})$, the SIFs should be different along the thickness of the crack tip. We adopted the conventional finite element method, and a three-dimensional seam $\left(\mathrm{SEAM}^{3 \mathrm{D}}\right)$ crack model was established, and the mesh near the crack tip was refined. The new extended finite element method (XFEM) (Golewski et al., 2012; Zhang and Bui, 2015; Roth et al., 2015) was applied, and a three-dimensional XFEM crack model without mesh refinement near the crack tip was established. Here, a comparison study is performed between the SIF calculated by the two-dimensional model (simplifying the three-dimensional problem into a planar problem) and the SIF calculated by the three-dimensional model to investigate the difference. Since XFEM cannot be used to calculate SIFs in two dimensions, the traditional finite element method was employed, and a two-dimensional seam crack model $\left(\mathrm{SEAM}^{2 \mathrm{D}}\right)$ with mesh refinement near the crack tip was established.

The meshes of the three models are shown in Fig. 8, and the mesh size was mainly set to $5 \mathrm{~mm}$. In the two-dimensional $\mathrm{SEAM}^{2 \mathrm{D}}$ model, the 6-node triangular element CPS6 was applied 
in the vicinity of the crack tip, and the 8-node quadrilateral element CPS8 was employed in the rest zone. In the three-dimensional SEAM ${ }^{3 \mathrm{D}}$ model, the 6-node linear triangular prism element C3D6 was used in the vicinity of the crack tip, and the 8-node linear brick element C3D8R (reduced integration, hourglass control) was applied in the rest zone.
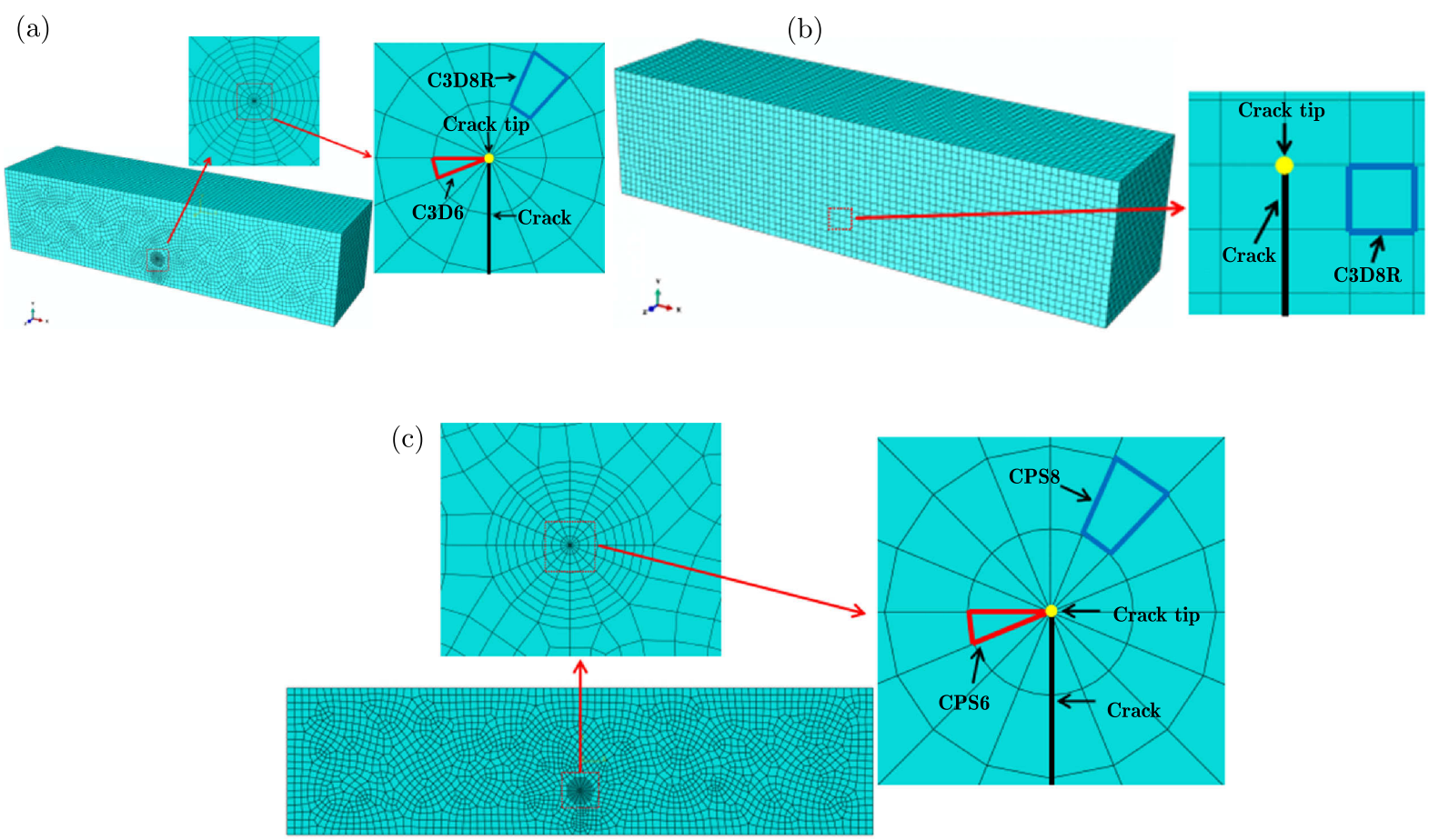

Fig. 8. Meshes of the anti-symmetrical four-point bending specimen in different numerical models:
(a) $\mathrm{SEAM}^{3 \mathrm{D}}$, (b) XFEM ${ }^{3 \mathrm{D}}$, (c) $\mathrm{SEAM}^{2 \mathrm{D}}$

XFEM is a new crack simulation method introduced by the ABAQUS code. The crack path does not depend on the element boundary, but it can be described by using the enrichment finite elements. Therefore, the crack obtained by XFEM can pass through the whole element. ABAQUS version 6.14 is utilized in this paper, which could support fracture flow, improve the judgment method of crack initiation, support non-local stress field to determine the direction of crack propagation and support the contour integral of the static XFEM crack. XFEM can be utilized to simulate propagation of the static crack and calculate the contour integral in the vicinity of the crack tip, in which the influence of residual stress could be considered.

\section{Results and discussion}

The experiment of anti-symmetrical four-point bending was conducted using a universal testing machine, and pure mode II stress intensity factors were calculated by using the ABAQUS code.

Taking one concrete specimen subjected to anti-symmetrical four-point bending as an example, the plot of the total force versus displacement is presented in Fig. 9. Then we can see what is the evolution of the stress intensity factor during the process of reaching the critical value.

The stress intensity factor is mainly used for brittle behavior of metals in specific fracture applications. For brittle materials like concrete, damage and failure constitutive models are mainly used, which are connected with typical failure criteria like Drucker-Prager, Lubliner etc. in Eq. (5.1) 1 and (5.1) $)_{2}$ (Jankowiak and Łodygowski, 2010; Xu, 2011). In addition, the stress intensity factor is connected with strain energy density in the notch region, see Eq. $(5.1)_{2}$ and $(5.1)_{3}$ 


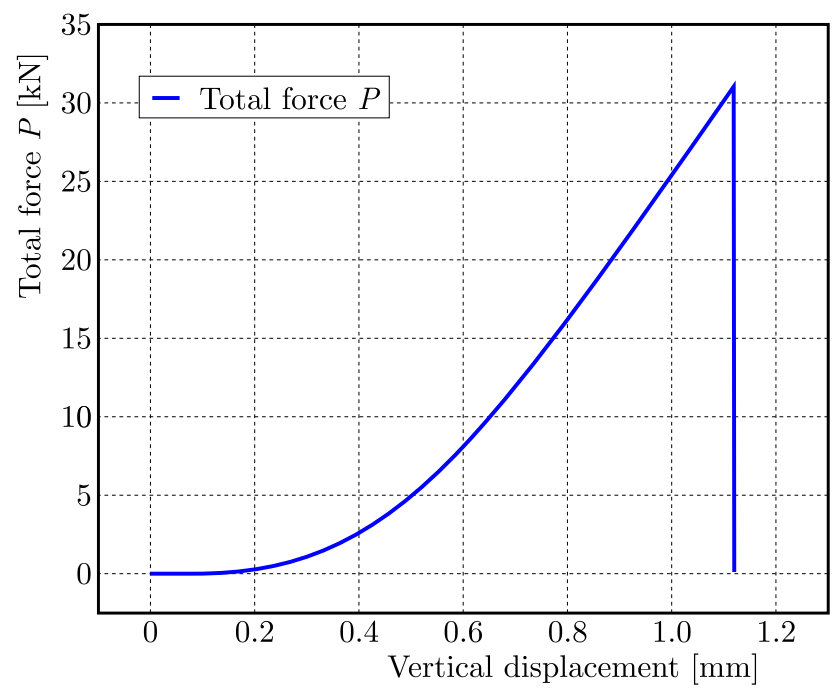

Fig. 9. Curve of the total force versus displacement

$$
f\left(I_{1}, I_{2}, I_{3}\right)=0 \quad \sigma_{i j}=\frac{K}{\sqrt{2 \pi r}} f_{i j}(\theta) \quad I_{1}=\sqrt{\frac{6 E W_{1}}{1-2 \nu}}
$$

where $I_{1}$ denotes the first invariant of the stress tensor $\sigma_{i j}, J_{2}$ and $J_{3}$ denote the second and third invariant of the stress deviator $s_{i j}$, respectively, $K$ denotes the stress intensity factor, $r$ and $\theta$ denote the polar coordinates in the vicinity of crack tip, $f_{i j}(\theta)$ denotes the direction parameter of $\theta, E$ and $\nu$ denote Young's modulus and Poisson's ratio, respectively, $W_{1}$ denotes the strain energy density of the volume change.

\subsection{Variation of SIF values along specimen thickness}

For three-dimensional problems, the stress intensity factor (SIF) may vary along the specimen thickness. In this study, the SIFs were calculated by using XFEM $^{3 \mathrm{D}}$ and SEAM ${ }^{3 \mathrm{D}}$, respectively, and the results of SIF values along the specimen thickness, i.e. $Z$-direction, are presented in Fig. 10. It can be seen that the SIFs $K_{I}, K_{I I}$ and $K_{I I I}$ obtained by both methods vary along the specimen thickness; the SIFs $K_{I}$ and $K_{I I I}$ are very small comparing to $K_{I I}$ values. This indicates that the crack mode is closest to pure mode II, especially near the center of the specimen thickness. Therefore, the SIF should be determined at the center of the specimen thickness.

It can be seen that the variation of $K_{I I}$ values calculated by $\mathrm{SEAM}^{3 \mathrm{D}}$ is larger than that by $\mathrm{XFEM}^{3 \mathrm{D}}$. In the calculation results by $\mathrm{SEAM}^{3 \mathrm{D}}$ in Fig. 10a, one can find that both $K_{I}$ and $K_{I I I}$ are close to zero near the specimen thickness center, whereas, for in results by XFEM ${ }^{3 \mathrm{D}}$ in Fig. 10b, only $K_{I I I}$ is close to zero, and $K_{I}$ is greater than $0.1 \mathrm{MPa} \sqrt{\mathrm{m}}$. This indicates that the calculation results by SEAM ${ }^{3 \mathrm{D}}$ are much closer to the real values of SIF than those by XFEM ${ }^{3 \mathrm{D}}$. Therefore, the SIF at the specimen thickness center will be analyzed in the subsequent study.

\subsection{SIF values on different integration contours in the specimen thickness center}

The SIF values may vary with integration contours. In this study, the SIFs with different integration contours were calculated by the aforementioned three models, and the results are presented in Fig. 11. One can find that the SIFs by SEAM ${ }^{2 \mathrm{D}}$ in Fig. 11a are stable, and $K_{I}$ and $K_{I I}$ are kept constant as the integration contour number increases. The SIFs by SEAM ${ }^{3 \mathrm{D}}$ in Fig. 11b show that as the integration contour number is 1 , the $K_{I I}$ value is the smallest, and as the contour number is 3 , the $K_{I I}$ value is the highest. As the contour number is larger than 3 , the $K_{I I}$ value decreases with the increase of the contour number, but the $K_{I}$ and $K_{I I I}$ 
(a)

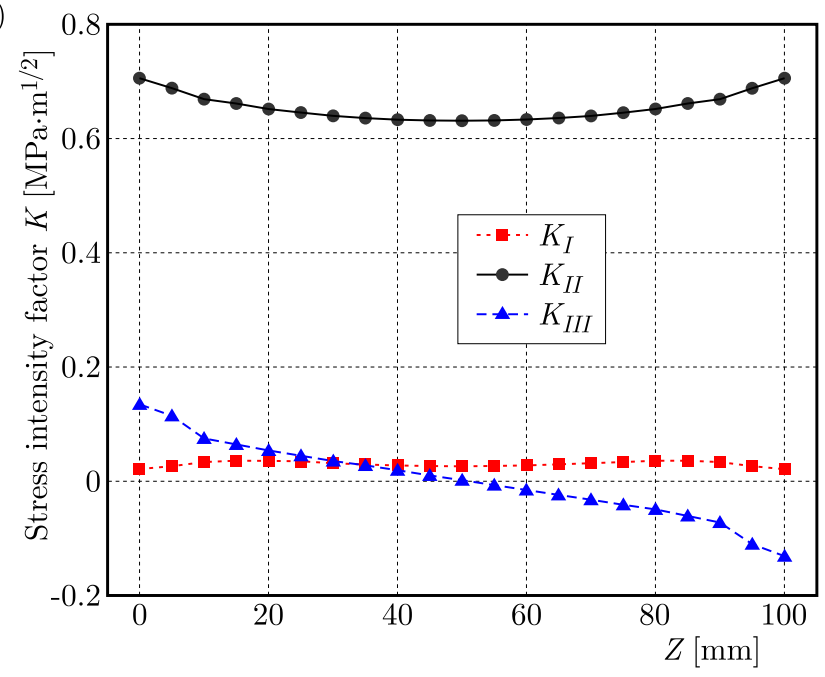

(b)

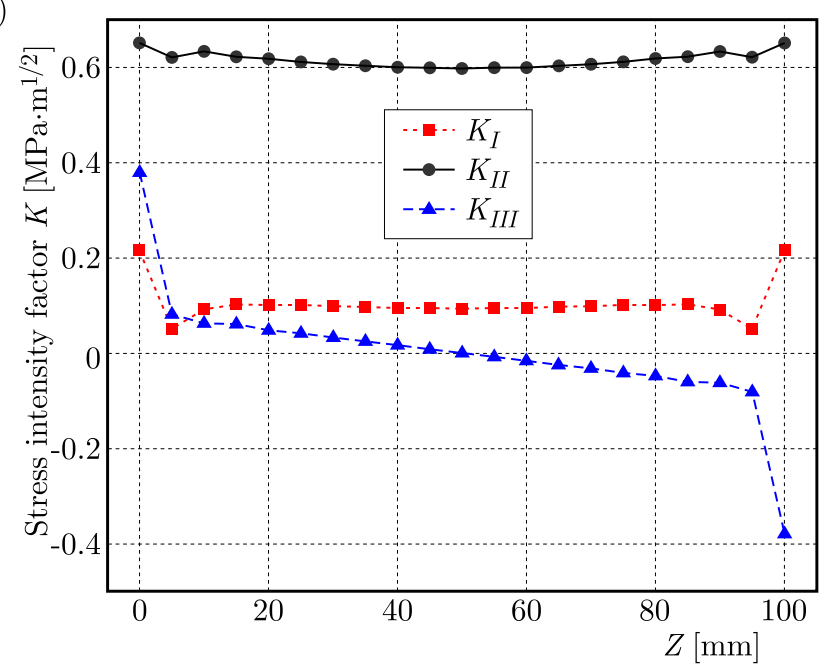

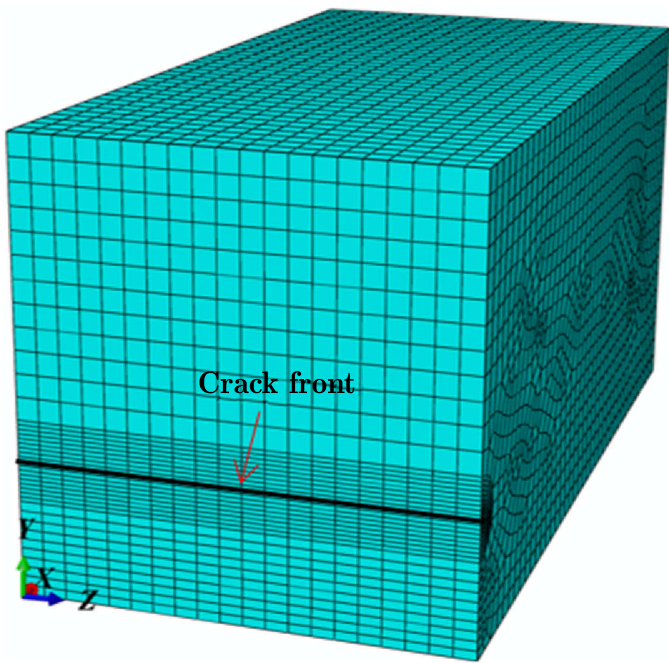

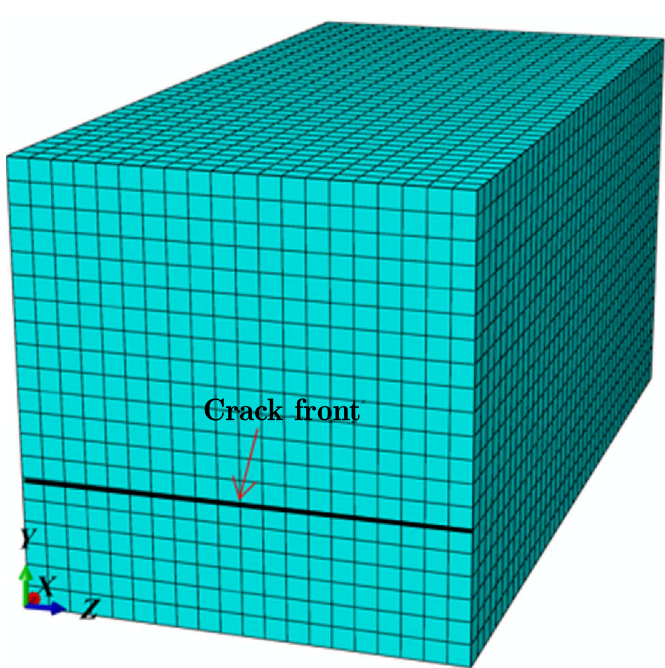

Fig. 10. Variation of SIFs in $Z$-direction: (a) $\mathrm{SEAM}^{3 \mathrm{D}}$, (b) XFEM ${ }^{3 \mathrm{D}}$

are kept constant. The SIFs by XFEM ${ }^{3 \mathrm{D}}$ in Fig. 11c show that all the $K_{I}$ and $K_{I I}$ values vary significantly with the contour numbers, but $K_{I I I}$ is constantly zero.

$K_{I I}$ results by the three methods are compared in Fig. 11d. It can be seen that the results by the $\mathrm{SEAM}^{2 \mathrm{D}}$ are stable, and the results by the SEAM ${ }^{3 \mathrm{D}}$ vary slightly, but the results by the $\mathrm{XFEM}^{3 \mathrm{D}}$ vary largely, and the maximum difference between the $K_{I I}$ values by 8-contour and by 9 -contour is $46 \%$. Therefore, the $\mathrm{XFEM}^{3 \mathrm{D}}$ should not be used to calculate SIF values.

Generally, the calculation results of $K_{I I}$ by $\mathrm{SEAM}^{3 \mathrm{D}}$ are less than those by $\mathrm{SEAM}^{2 \mathrm{D}}$, and when the integration contour is in the specimen thickness center, the difference between these two methods is $1.371 \%$.

\subsection{SIF values for different mesh refinement circle numbers in the specimen thickness center}

Taking SEAM ${ }^{3 \mathrm{D}}$ as an example, one, three, six and nine circle models are designed to investigate the influence of mesh refinement circles on calculating mode II SIF. The circles distribution is shown in Fig. 12, where mode II SIF is determined at the center of the specimen thickness.

Nine contours enclosing the crack tip are designed to calculate pure mode II SIF using the ABAQUS code. In the light of SIFs on the innermost and outermost contour, they fluctuate 
(a)

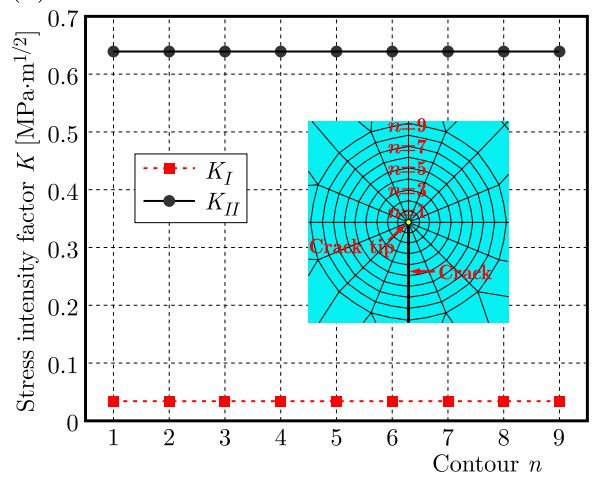

(c)

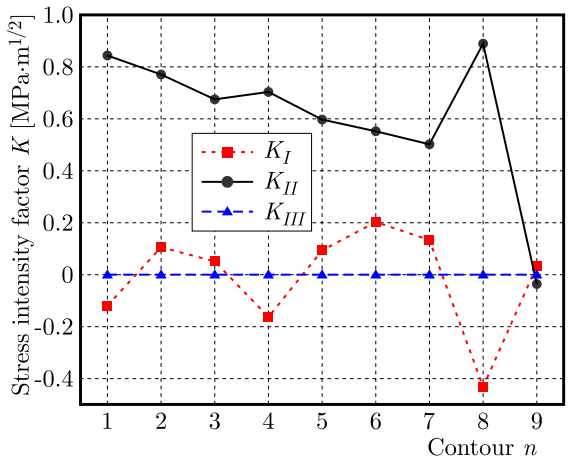

(b)
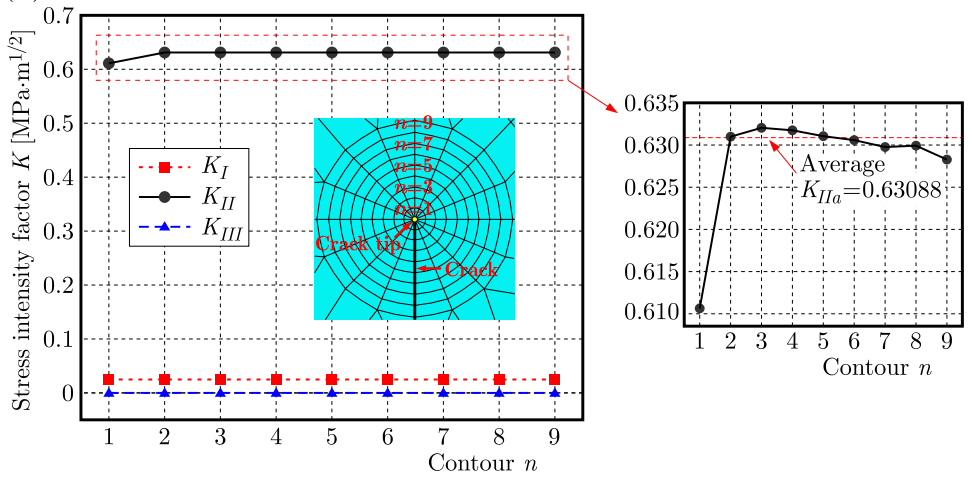

(d)
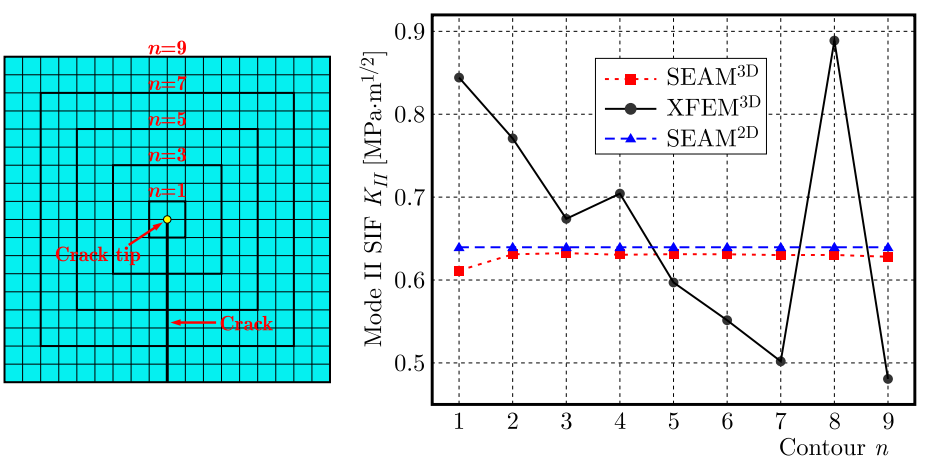

Fig. 11. SIFs on different contours using three numerical models: (a) SEAM ${ }^{2 D}$, (b) SEAM ${ }^{3 \mathrm{D}}$, (c) $\mathrm{XFEM}^{3 \mathrm{D}}$, (d) comparison

(a)

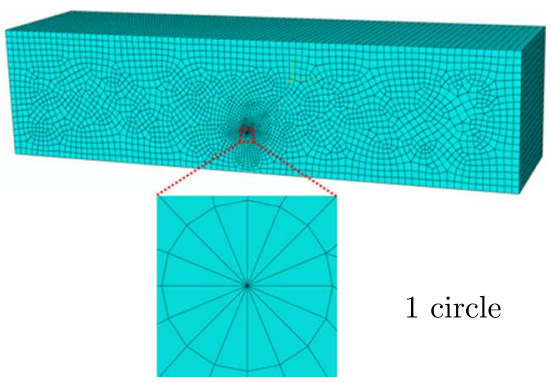

(c)

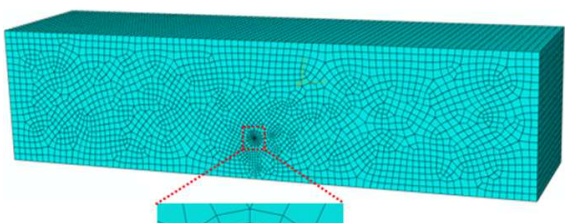

6 circle (b)

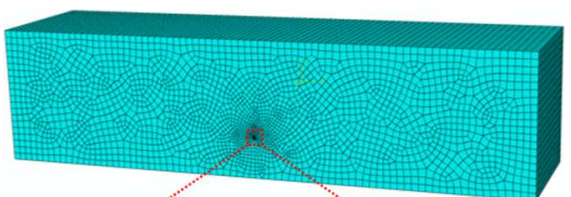

3 circle

(d)

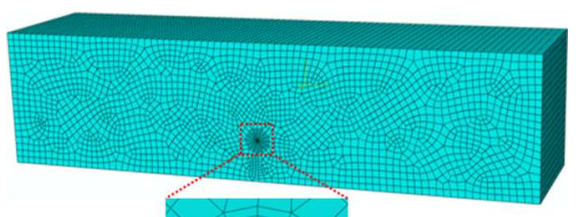

9 circle

Fig. 12. Nine and three integration circle contours in the vicinity of the crack tip $\left(\mathrm{SEAM}^{3 \mathrm{D}}\right)$ : (a) 1 circle contour, (b) 3 circle contours, (c) 6 circle contours, (d) 9 circle contours

considerably, and were discarded, and the average of SIFs on the rest seven contours is taken as $K$. The analysis results are revealed in Fig. 13. It can be observed that mode II SIFs calculated by the one, three and six circle models fluctuate considerably. However, the SIFs calculated by the nine circle model fluctuate slightly, and the SIF is determined by the average of SIFs on 
the rest seven contours. The average mode II SIFs are present in Table 4. The maximum and minimum mode II SIFs $K_{I I}$ are $0.6309 \mathrm{MPa} \sqrt{\mathrm{m}}$ and $0.6107 \mathrm{MPa} \sqrt{\mathrm{m}}$ using 1 circle and 9 circle models, respectively. The maximum difference between these circle models is $2.052 \%$.

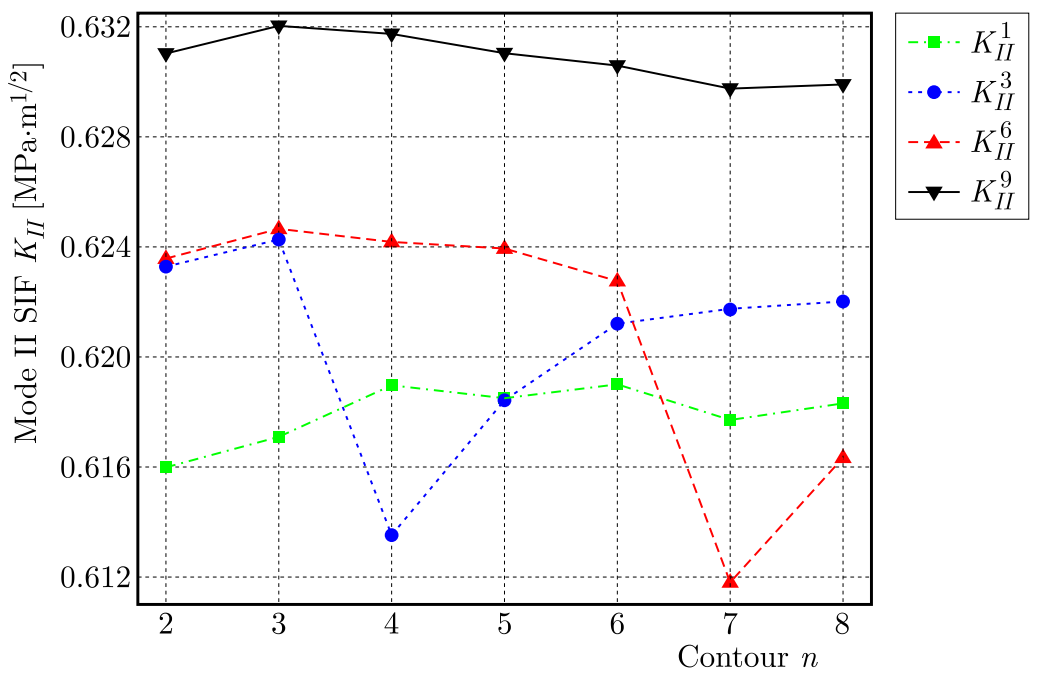

Fig. 13. Mode II SIFs $K_{I I}$ varying along contour $n$ for different mesh refinement circles in the vicinity of the crack tip $\left(\mathrm{SEAM}^{3 \mathrm{D}}\right)$

Table 4. Average mode II stress intensity factor $K_{I I}$ for different number of circles

\begin{tabular}{|c|c|c|c|c|}
\hline Number of circles & 1 & 3 & 6 & 9 \\
\hline \hline Average mode II SIF $K_{I I}[\mathrm{MPa} \sqrt{\mathrm{m}}]$ & 0.6179 & 0.6209 & 0.621 & 0.6309 \\
\hline
\end{tabular}

\subsection{Verification of meshing independency}

Five mesh sizes ( $1 \mathrm{~mm}, 3 \mathrm{~mm}, 5 \mathrm{~mm}, 7 \mathrm{~mm}$ and $10 \mathrm{~mm}$ ) are selected to investigate the effect of mesh size on the calculation of SIFs. The mesh models are shown in Fig. 14. There is little difference (about $1.371 \%$ gap) between SIFs calculated by the two-dimensional and three-dimensional model in the conventional computation of SIFs. In order to improve the calculation efficiency and considerably reduce the amount of computation, the two-dimensional model is applied to verify the mesh independency. The number of elements and the average SIF in different models are illustrated in Table 5. It can be found that there is almost no difference for pure mode SIF $K_{I I}$ for different mesh size. When the mesh size decreases, the number of elements increases enormously, which increases the amount of calculation considerably. The meshing independence of these models is pretty good. Here we select a model with medium mesh size of $5 \mathrm{~mm}$, the calculation results are accurate, the number of elements is moderate, and the calculation amount is acceptable.

Table 5. Number of elements and pure mode II stress intensity factor $K_{I I}$ for different element sizes

\begin{tabular}{|c|c|c|c|c|c|}
\hline Mesh size $[\mathrm{mm}]$ & 1 & 3 & 5 & 7 & 10 \\
\hline \hline Element number & 49206 & 5356 & 2293 & 1307 & 761 \\
\hline$K_{I I}[\mathrm{MPa} \sqrt{\mathrm{m}}]$ & 0.6396 & 0.6396 & 0.6395 & 0.6396 & 0.6394 \\
\hline
\end{tabular}


(a)

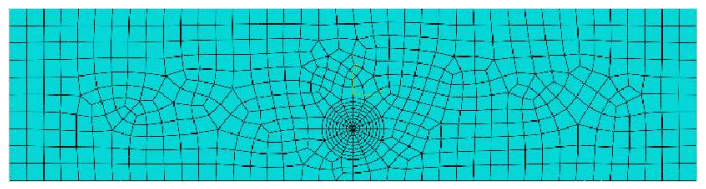

(c)

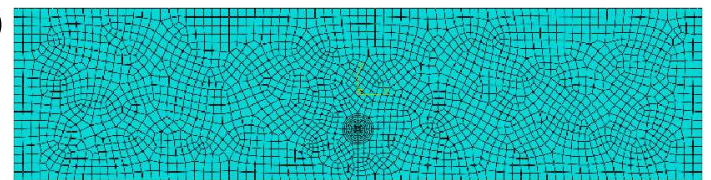

(b)

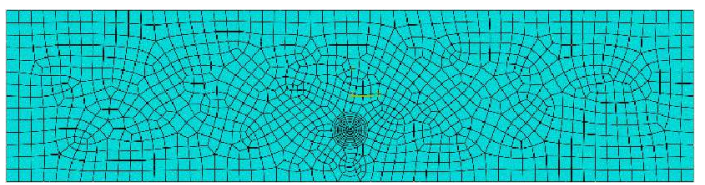

(d)

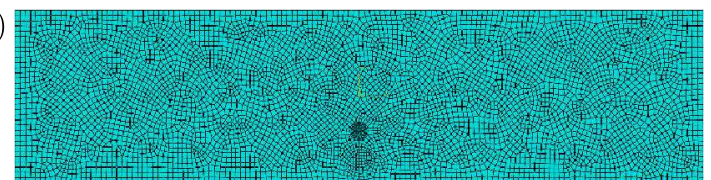

(e)

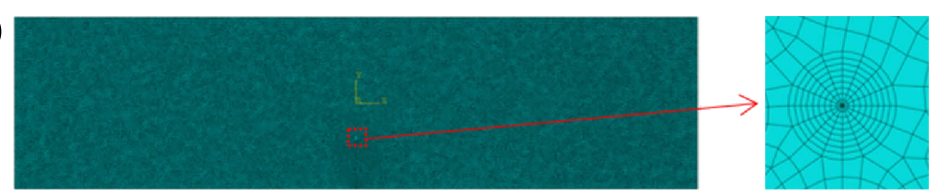

Fig. 14. Model meshes for different element size $\left(\mathrm{SEAM}^{2 \mathrm{D}}\right)$; mesh size: (a) $10 \mathrm{~mm}$, (b) $7 \mathrm{~mm},(\mathrm{c}) 5 \mathrm{~mm}$, (d) $3 \mathrm{~mm}$, (e) $1 \mathrm{~mm}$

\section{Conclusions}

In this paper, pure mode II stress intensity factors of fine-grained concrete were investigated by performing anti-symmetrical four-point bending experimentally and numerically. The following conclusions are obtained:

- In the three-dimensional numerical models, the crack mode is closest to pure mode II at the center of specimen thickness.

- Pure mode II stress intensity factor obtained by $\mathrm{SEAM}^{2 \mathrm{D}}$ and $\mathrm{XFEM}^{3 \mathrm{D}}$ is $1.36 \%$ and $6.17 \%$ respectively larger than that by $\mathrm{SEAM}^{3 \mathrm{D}}$.

- Pure mode II stress intensity factors of concrete obtained by the conventional finite element method (FEM) has better stability than that by XFEM.

- The number of mesh refinement circles has slight influence on the calculation of pure mode II stress intensity factor.

\section{Funding}

This work was financially supported by the National Natural Science Foundation of China (grant number U19A2098), the Sichuan Science and Technology Program (grant number 2021YJ0511), the Key Laboratory of Rock Mechanics and Geohazards of Zhejiang Province (grant number ZJRMG-202001), the Open fund of Key Laboratory of Deep Underground Science and Engineering (grant number DESE202005), the Fundamental Research Funds for the Central Universities.

\section{References}

1. ABAQUS HTML Documentation, 2016, Dassault Systemes

2. Braham A., Buttlar W., 2009, Mode II cracking in asphalt concrete, Advanced Testing and Characterization of Bituminous Materials, 2, 699-706

3. Dong W., Wu Z., Tang X., Zhou X., 2018, A comparative study on stress intensity factor-based criteria for the prediction of mixed mode I-II crack propagation in concrete, Engineering Fracture Mechanics, 197, 217-235

4. Fett T., 1999, Stress intensity factors for edge-cracked plates under arbitrary loading, Fatigue Fracture of Engineering Materials and Structures, 22, 4, 301-305 
5. Golewski G.L., Golewski P., Sadowski T., 2012, Numerical modelling crack propagation under mode II fracture in plain concretes containing siliceous fly-ash additive using XFEM method, Computational Materials Science, 62, 75-78

6. He M.Y., CaO H.C., Evans A.G., 1990, Mixed-mode fracture: the four-point shear specimen, Acta Metallurgica et Materialia, 38, 5, 839-846

7. IRWIN G.R., 1957, Analysis of stresses and strains near the end of a crack traversing a plate, Journal of Applied Mechanics, 24, 361-364

8. Jankowiak T., Łodygowski T., 2010, Quasi-static failure criteria for concrete, Archives of Civil Engineering, 56, 2, 123-154

9. Kaplan M.F., 1961, Crack propagation and the fracture of concrete, Journal Proceedings, 58, 11, 591-610

10. Orowan E., 1949, Fracture and strength of solids, Reports on Progress in Physics, 12, 1, 185

11. RiCE J.R., 1968, A path independent integral and the approximate analysis of strain concentration by notches and cracks, Journal of Applied Mechanics, 35, 2, 379-386

12. Roth S.N., LÉGer P., Soulä̈mani A., 2015, A combined XFEM-damage mechanics approach for concrete crack propagation, Computer Methods in Applied Mechanics and Engineering, 283, 923-955

13. Shin C.F., Moran B., Nakamura T., 1986, Energy release rate along a three-dimensional crack front in a thermally stressed body, International Journal of Fracture, 30, 2, 79-102

14. Stys D., Minch M., 1993, Influence of mode II stress intensity factor on the fracture toughness of concrete, International Conference on Fracture ICF8, Kiev, Ukraine

15. WAng C., Zhu Z.M., LiU H.J., 2016, On the I-II mixed mode fracture of granite using four-point bend specimen, Fatigue Fracture of Engineering Materials and Structures, 39, 10, 1193-1203

16. Wittmann F.H., Roelfstra P.E., Mihashi H., Huang Y.Y., Zhang X.H., Nomura N., 1987, Influence of age of loading, water-cement ratio and rate of loading on fracture energy of concrete, Materials and Structures, 20, 2, 103-110

17. Wu Y., Chen H., Wang X., 2017, Numerical flexibility determination method of stress intensity factor for concrete, Materials Science and Engineering Conference Series, 250, 1, 012040

18. Xu S.L., 2011, Fracture Mechanics of Concrete (in Chinese), Science Press, Beijing

19. Zhang X., Bui T.Q., 2015, A fictitious crack XFEM with two new solution algorithms for cohesive crack growth modeling in concrete structures, Engineering Computations, 32, 2, 473-497

20. Zhang X.X., Ruiz G., Yu R.C., TARifa M., 2009, Fracture behavior of high-strength concrete at a wide range of loading rates, International Journal of Impact Engineering, 36, 10-11, 1204-1209

21. Zhou C., Zhu Z., 2019a, Study of crack dynamic propagation behavior of fine-grained concrete under static loading, International Journal of Fracture, 220, 113-125

22. Zhou C., Zhu Z., Wang Z., QIU H., 2018, Deterioration of concrete fracture toughness and elastic modulus under simulated acid-sulfate environment, Construction and Building Materials, 176, 490-499

23. Zhou C., Zhu Z., Zhu A., Zhou L., Fan Y., LAng L., 2019b, Deterioration of mode II fracture toughness, compressive strength and elastic modulus of concrete under the environment of acid rain and cyclic wetting-drying, Construction and Building Materials, 228, 116809 